\title{
Two-dimensional self-assembly and electrical properties of the donor-acceptor tetrathiafulvalene-polychlorotriphenylmethyl radical on graphite substrates $\oplus^{\oplus}$
}

Cite as: J. Appl. Phys. 125, 142909 (2019); https://doi.org/10.1063/1.5065448

Submitted: 10 October 2018 . Accepted: 29 November 2018. Published Online: 02 April 2019

Judith Guasch (D), Núria Crivillers (D), Manuel Souto (D), Imma Ratera (D), Concepció Rovira (D), Paolo Samorì (iD), and Jaume Veciana (iD)

\section{COLLECTIONS}

Paper published as part of the special topic on Molecular Spintronics

EP This paper was selected as an Editor's Pick
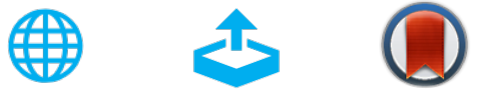

\section{ARTICLES YOU MAY BE INTERESTED IN}

A multifunctional molecular spintronic platform with magnetoresistive and memristive responses via a self-assembled monolayer

Journal of Applied Physics 125, 142905 (2019); https://doi.org/10.1063/1.5057893

Molecular discovery of half-metallic one-dimensional metal-organic framework Journal of Applied Physics 125, 142906 (2019); https://doi.org/10.1063/1.5079421

Studies of spin transport in fullerene films

Journal of Applied Physics 125, 142908 (2019); https://doi.org/10.1063/1.5079440

Lock-in Amplifiers Find out more today

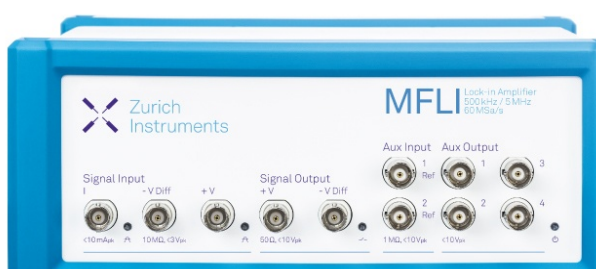

Online 


\title{
Two-dimensional self-assembly and electrical properties of the donor-acceptor tetrathiafulvalene-polychlorotriphenylmethyl radical on graphite substrates
}

Cite as: J. Appl. Phys. 125, 142909 (2019); doi: 10.1063/1.5065448

Submitted: 10 October 2018 . Accepted: 29 November 2018 .

Published Online: 2 April 2019

Judith Guasch, ${ }^{1, a)}$ (D) Núria Crivillers, ${ }^{1,2}$ (D) Manuel Souto, ${ }^{1, b)}$ (D) Imma Ratera, ${ }^{1}$ (D) Concepció Rovira, ${ }^{1}$ (D) Paolo Samori, ${ }^{2}$ (iD and Jaume Veciana ${ }^{1, a)}$ (D)

\author{
AFFILIATIONS \\ ${ }^{1}$ Institut de Ciència de Materials de Barcelona (CSIC)/CIBER-BBN, Campus UAB, Cerdanyola del Vallès, 08193 Barcelona, Spain \\ ${ }^{2}$ Université de Strasbourg, CNRS, ISIS UMR 7006, 8 allée Gaspard Monge, 67000 Strasbourg, France

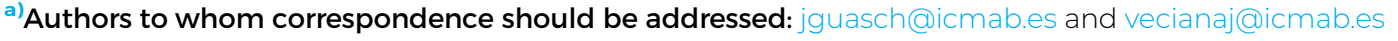 \\ b)Present address: Instituto de Ciencia Molecular (ICMol), Universidad de Valencia, Catedrático José Beltrán 2, 46980 Paterna, \\ Spain.
}

\begin{abstract}
The electron donor-acceptor tetrathiafulvalene-polychlorotriphenylmethyl (PTM) radical dyad, which shows a strong interplay between intra- and intermolecular charge transfer processes in solution, has been deposited by drop-casting on highly oriented pyrolytic graphite substrates, and its self-assembled structure has been investigated. Conducting atomic force microscopy revealed that the presence of a PTM radical in the molecules enhances the electrical conduction by almost two orders of magnitude and that this enhancement occurs in spite of the poor molecular orientation control achieved with drop-casting. Moreover, the study also reveals that the presence of a tetrathiafulvalene subunit in the deposited molecules can result in slightly asymmetric I-V curves.
\end{abstract}

Published under license by AIP Publishing. https://doi.org/10.1063/1.5065448

\section{INTRODUCTION}

The rapid strides being made in the miniaturization of electronic components are triggering new research interest focused on the development of new electro-active supramolecular structures as active elements for the realization of molecular-based devices such as switches, memories, and diodes. ${ }^{1,2}$ Organic donor-acceptor (D-A) dyads are very attractive systems to produce molecular diodes as building blocks for logic circuitries. ${ }^{3}$ Indeed, physisorbed Langmuir-Blodgett (LB) films based on the D-A dyad hexadecylquinolinium tricyanoquinodimethanide embedded between metallic contacts displayed asymmetric current-voltage (I-V) plots and thus it was proposed as the first molecular rectifier. ${ }^{4-8}$ Further, LB films of D-A dyads with enhanced donor or acceptor properties, containing, for example, tetrathiafulvalene (TTF) or fullerene subunits, were also prepared. ${ }^{9,10}$ In some of them, it was demonstrated that the displayed rectification characteristics were caused only by the chemical structure of the molecules and not by their specific orientation, ${ }^{11-13}$ or by Schottky barriers produced by the use of different contacts. ${ }^{14}$ Indeed, the molecular self-assembly on a given solid substrate and the method to wire it up are two crucial factors to be considered when thinking about future applications in molecular electronics. The deposition of molecules from the liquid phase to a substrate by drop-casting is an extremely easy, cheap, and fast method. ${ }^{15}$ Furthermore, it has the advantage that large molecules can be processed without decomposition and sometimes without the need of being functionalized with specific side-groups. Nevertheless, such a physical adsorption process usually yields random molecular orientations and limited order at the supramolecular level within the films. This result can be ascribed to the interplay of different factors such as the limited flatness at the atomic scale of the metal electrodes, the variable molecular surface, and molecule-molecule interactions as well as dewetting 
<smiles>Clc1c(Cl)c(Cl)c(C(c2c(Cl)c(Cl)c(Cl)c(Cl)c2Cl)c2c(Cl)c(Cl)c(/C=C/C3=CSC(=C4SC=CS4)S3)c(Cl)c2Cl)c(Cl)c1Cl</smiles><smiles>Clc1cc(C=CC2=CSC(=C3SC=CS3)S2)c(Cl)c(Cl)c1C(c1c(Cl)c(Cl)c(Cl)c(Cl)c1Cl)(c1c(Cl)c(Cl)c(Cl)c(Cl)c1Cl)c1c(Cl)c(Cl)c(Cl)c(Cl)c1Cl</smiles><smiles>Clc1c(Cl)c(Cl)c(C(c2c(Cl)c(Cl)c(Cl)c(Cl)c2Cl)c2c(Cl)c(Cl)c(Cl)c(Cl)c2Cl)c(Cl)c1Cl</smiles>

SCHEME 1. TTF-PTM radical dyad 1 , non-radical analogue dyad 2 , and PTM radical 3.

phenomena. To improve this, a number of experimental parameters can be optimized. For example, ultraflat conducting surfaces can be used as electrodes such as highly oriented pyrolytic graphite (HOPG) substrates or graphene. The use of such carbonaceous surfaces has two advantages. First, large atomically flat areas extending several hundreds of $\mu \mathrm{m}^{2}$ can be easily obtained due to the "millefeuille" structure, with weak van der Waals forces holding adjacent layers together. Secondly, aromatic hydrocarbons or molecular systems bearing long alkyl chains are prone to self-assemble on such substrates, forming highly ordered structures. ${ }^{16,17}$ For example, Bottari et al. ${ }^{18}$ reported the self-assembly of a D-A dyad consisting of phthalocyanine and fullerene $\left(\mathrm{Pc}_{-} \mathrm{C}_{60}\right)$ on HOPG which forms layers and fibers depending on the concentration of the drop-cast solution. The electrical properties of these selfassembled structures were investigated by conducting atomic force microscopy (C-AFM), a technique that allows one to combine morphological and electrical mapping of a sample with a spatial and electrical resolution of a few tens of nanometers and $\mathrm{pA}$, respectively. ${ }^{19}$ Interestingly, both $\mathrm{Pc}_{\mathrm{c}} \mathrm{C}_{60}$ supramolecular structures showed high conductivities comparable to the HOPG surface, yet not accompanied by electrical rectification. With the objective of attaining diode-like characteristics using different D-A dyads on graphite, we have investigated the electrical properties of the previously reported TTF-polychlorotriphenylmethyl radical (PTM) dyad 1 (Scheme 1), ${ }^{20-22}$ which is electro- and magnetically-active. It has been reported that radical dyad 1 shows a complex interplay between its intramolecular electron transfer (IET) from the donor (TTF) to the acceptor (PTM) ${ }^{21}$ moieties and its intermolecular charge transfer (CT) mediated by the TTF subunit, giving rise in solution to interesting supramolecular structures ${ }^{2,23}$ including, for example, a reversible switch between diamagnetic dimers, observed at room temperature, and paramagnetic monomers at high temperature. ${ }^{24}$

Here, we analyze the self-assembly of radical dyad $\mathbf{1}$ on HOPG substrates obtained by simple drop-casting through AFM imaging. The results obtained have been compared with those observed when processing the non-radical analogue dyad 2 and the unsubstituted PTM radical 3 (Scheme 1) into similar films.

The chemical structure of dyad $\mathbf{2}$ differs from $\mathbf{1}$ only in one hydrogen atom, which is linked to the central carbon atom of the PTM subunit causing a hybridization change $\left(s p^{3}\right.$ and $s p^{2}$, respectively), thereby nullifying the radical character and decreasing the electron acceptor strength of the PTM subunit. Thus, the comparison between 1 and 2 might shed light on the influence of the radical character on the properties. On the other hand, the surfaces drop-cast with radical compound 3 could give an insight into the role of the TTF aggregation phenomena, i.e., the CT process. The electrical properties of these films were investigated by C-AFM, revealing that the presence of the PTM radical is needed to obtain a significant conduction, whereas the TTF subunit causes the appearance of slightly asymmetric I-V curves, i.e., a very modest electrical rectification.

\section{SELF-ASSEMBLY OF THE TTF-PTM RADICAL DYAD ON HOPG}

Figure 1 displays AFM tapping mode images of surfaces prepared by drop-casting $(2 \times 25 \mu \mathrm{l})$ diluted solutions $(0.01 \mathrm{mM})$ of the radical dyad 1 in DMF (S1), $\mathrm{CH}_{2} \mathrm{Cl}_{2}$ (S2), and toluene (S3) onto freshly cleaved HOPG surfaces. The depositions were performed under atmospheric conditions and in the dark to avoid the decomposition of the radical. Radical dyad 1 presented the highest surface coverage when DMF was the solvent employed (S1). Such results can be explained by the higher affinity of the dyad to interact with the substrate rather than with neighboring molecules forming large stacks. Indeed, the high boiling point $\left(153^{\circ} \mathrm{C}\right)$ and low volatility of DMF favors the formation of thermodynamically stable structures, offering to the molecules enough time to organize on the substrate. In contrast, when solvents with lower boiling points, such as toluene $\left(111^{\circ} \mathrm{C}\right)$ and $\mathrm{CH}_{2} \mathrm{Cl}_{2}\left(40^{\circ} \mathrm{C}\right)$, are used, the 

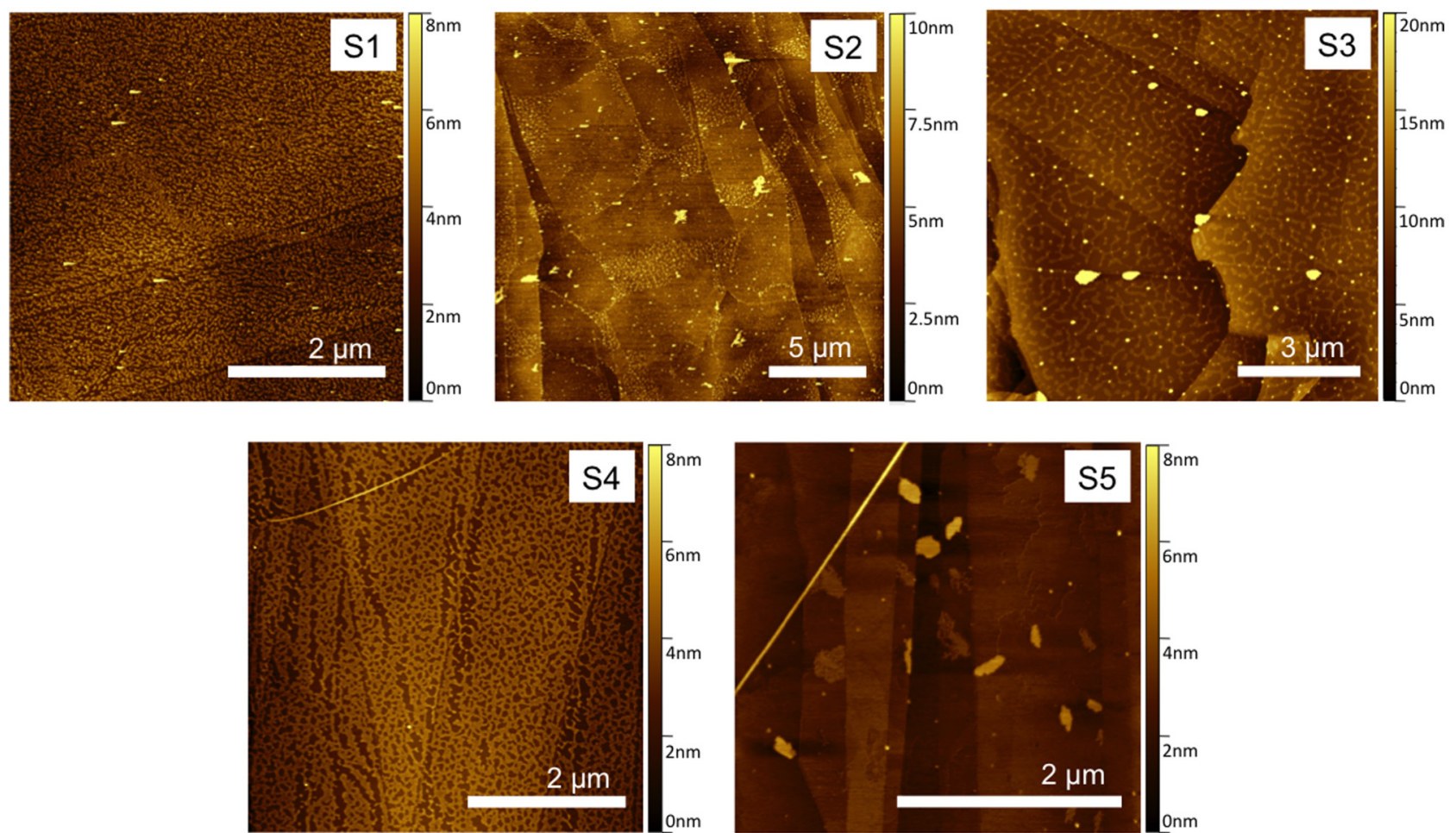

FIG. 1. Tapping-mode AFM topographic images of the different modified graphite substrates obtained by drop-casting the three different compounds using $0.01 \mathrm{mM}$ solutions (S1: 1/DMF; S2: 1/toluene; S3: $1 / \mathrm{CH}_{2} \mathrm{Cl}_{2}$; S4: 2/DMF; S5: 3/DMF).

molecular self-assembly process does not reach the thermodynamic minimum due to the faster solvent evaporation (S2 and S3, respectively), ultimately resulting in rather disordered assemblies. In fact, this phenomenon can be even exploited to pattern substrates through the dewetting phenomena (Fig. S1 in the supplementary material). ${ }^{25,26}$ Consequently, we analyzed the nanostructures formed by the non-radical dyad $\mathbf{2}$ and radical $\mathbf{3}$ in DMF ( $\mathbf{S 4}$ and S5, respectively). Compound $\mathbf{3}$ forms isolated plate-like structures, with lateral sizes of several nanometers, in comparison with the assemblies of the TTF-containing molecules $\mathbf{1}$ and 2, which exhibit more continuous structures, especially when the PTM radical subunit is present. These structures are believed to be induced by the presence of the TTF subunit, which favors the interaction between molecules. ${ }^{27}$ It is also worth mentioning that all the observed structures display heights of 2-3 nm (Fig. S2 in the supplementary material), indicating that mono- or bilayer thick assemblies were formed according to the molecular length of the molecules.

We have then extended our study to the analysis of the magnetic and electrical properties of the modified HOPG substrates by using electron spin resonance (ESR) spectroscopy and cyclic voltammetry $(\mathrm{CV})$, with the goal of determining if the properties of radical dyad 1 were retained once they were deposited onto the substrates. The ESR spectrum of surface $\mathbf{S 1}$ performed at RT exhibits two different signals [Fig. S3(a) in the supplementary material]. The small one corresponds to the PTM radical subunit of dyad $\mathbf{1}$, whereas the large signal can be ascribed to the HOPG; i.e., it is an intrinsic signal of the surface. To avoid the overlapping of both signals, the surface $\mathbf{S 1}$ was placed inside the ESR instrument and rotated along the $\mathrm{z}$-axis because the $g$-value of the ESR signal related to the HOPG surface strongly depends on the angle between the surface and the applied magnetic field, whereas the signal of the PTM subunit remains practically constant due to its low magnetic anisotropy. Thus, we recorded three different spectra at different angles $\left(0^{\circ}, 45^{\circ}\right.$, and $\left.65^{\circ}\right)$ in order to clearly detect a signal at a $g$-value of 2.0039 and a linewidth of $\Delta \mathrm{H}_{\mathrm{pp}}=5.8 \mathrm{G}$, typical for physisorbed PTM radicals on HOPG surfaces. ${ }^{28}$ Our analysis confirmed that the radical character of dyad $\mathbf{1}$ is preserved upon physisorption on the HOPG surface, thus indicating that the magnetic properties of the molecule are unaltered. The CV of S1 was carried out by immersing the coated surface, which was used as the working electrode, in a $0.15 \mathrm{M}$ solution of the electrolyte $\left(n-\mathrm{Bu}_{4} \mathrm{NPF}_{6}\right)$ in dry acetonitrile. The counter electrode used was a platinum wire, whereas the reference electrode was $\mathrm{Ag} / \mathrm{AgCl}$ [Fig. S3(b) in the supplementary material]. The obtained voltammogram exhibits a couple of weak waves due to the low concentration of molecules on the surface and some unavoidable solubilization of the physisorbed molecule. However, two redox processes can be identified at $E_{1 / 2}^{\text {red }} \approx 0.023 \mathrm{~V}$ and $E_{1 / 2}^{o x 1} \approx 0.257 \mathrm{~V}$, which can be attributed to the $\mathrm{PTM}^{*} / \mathrm{PTM}^{-}$and the $\mathrm{TTF} / \mathrm{TTF}^{+}$ redox pairs of dyad $\mathbf{1}$, respectively. It is worth noting that it was 
a)

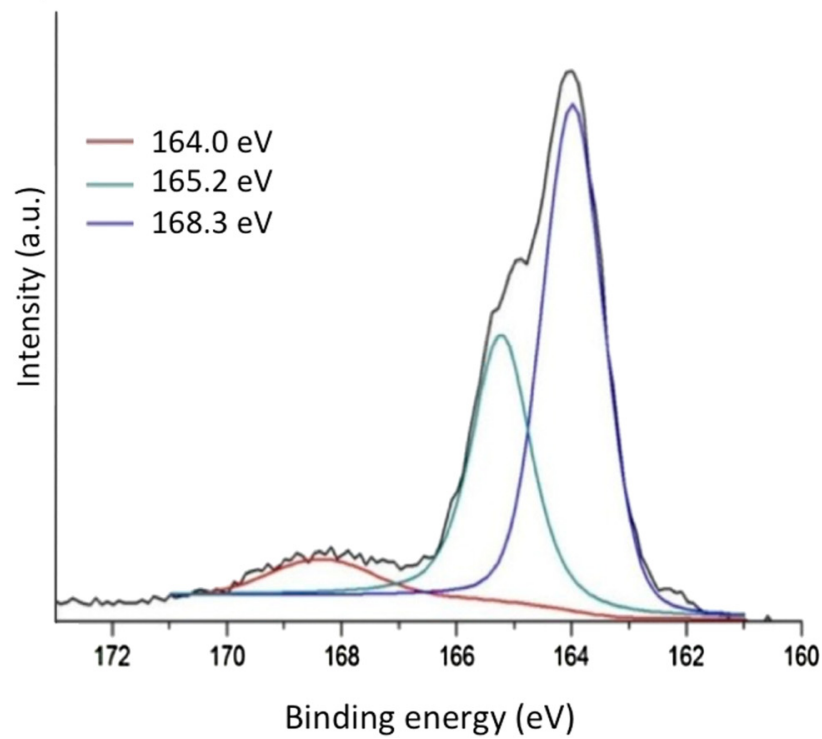

b)

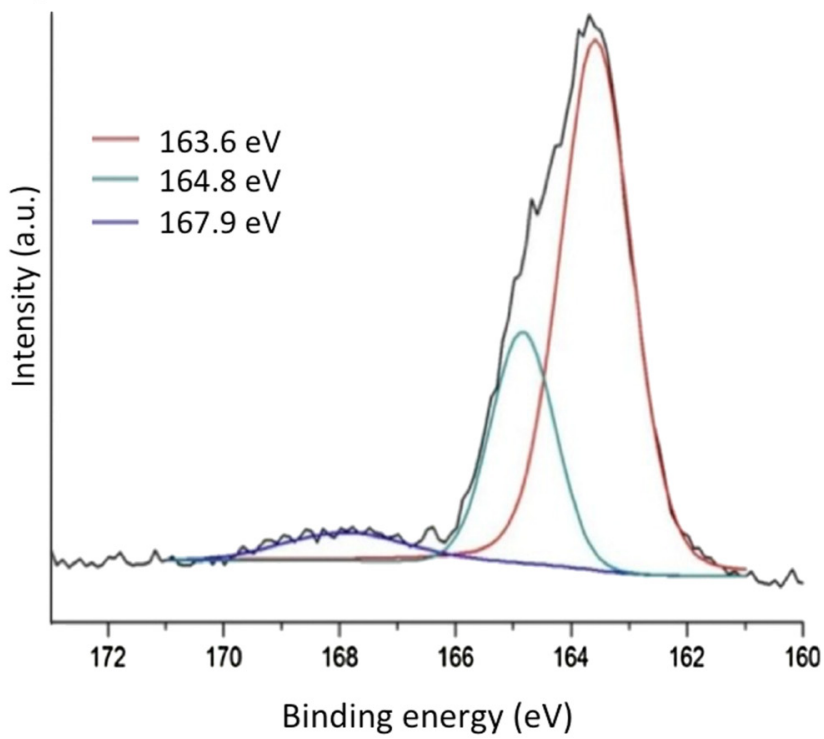

FIG. 2. High-resolution S2p XPS spectra of (a) $\mathbf{S 1}$ and (b) S4 surfaces.

not possible to detect the expected third wave associated with the $\mathrm{TTF}^{+} \bullet / \mathrm{TTF}^{2+}$ redox process at higher potentials.

To further study the electronic structure of compounds $\mathbf{1}$ and $\mathbf{2}$ deposited on HOPG substrates, X-ray photoelectron spectroscopy (XPS) was performed. Thus, high-resolution spectra for all the relevant elements $(\mathrm{S}, \mathrm{Cl}, \mathrm{C}, \mathrm{O})$ of both $\mathbf{S 1}$ and $\mathbf{S 4}$ substrates were recorded at RT. The binding energy of the $\mathrm{S} 2 p$ line gives information about the redox state of the TTF subunit (Fig. 2). Specifically, it is reported that the peak at $163.5 \mathrm{eV}$ is associated to a neutral TTF molecule, whereas the peak at $164.6 \mathrm{eV}$ corresponds to the positively charged $\mathrm{TTF}^{+\bullet}$ molecule. The S2p spectrum of S4 exhibits two peaks at 163.6 and $164.8 \mathrm{eV}$, which are ascribed to the neutral molecule as well as a charge transfer between the molecule and the HOPG substrate, as previously described for similar TTF-based structures. ${ }^{29}$ S1 also shows two peaks at 164.0 and $165.2 \mathrm{eV}$. In this case, the signal corresponding to $\mathrm{TTF}^{+}$could be related to both the molecular surface charge transfer and the presence of IET in dyad $\mathbf{1}$ stabilized by $\mathrm{DMF}^{20,22-24}$

To shed some light on this issue, temperature-dependent XPS spectra of S1 were performed at $+124,+25$, and $-174^{\circ} \mathrm{C}$ (Fig. S4 in the supplementary material). The absence of significant changes in the resulting spectra indicates that the presence of oxidized TTF possibly corresponds only to the molecular surface charge transfer. Similarly, temperature-dependent ESR spectra of dyad $\mathbf{1}$ in bulk (Fig. S5 in the supplementary material) only show a single line at $g=2.0033$ with a $\Delta \mathrm{H}_{\mathrm{pp}}=2.7 \mathrm{G}$, which is related to the PTM radical. Thus, dyad $\mathbf{1}$ is found in its neutral state unlike a similar radical dyad, which consists of a ferrocene subunit instead of a TTF subunit, the ferrocene-PTM dyad that presents bistability at the same range of temperatures $(300-120 \mathrm{~K}) .^{30-34}$ We also characterized the surface modified with the radical dyad 1 by time-of-flight secondary ion mass spectrometry (TOF-SIMS) using a concentrated solution ( $1 \mathrm{mM}$ ) in DMF, which yielded surface $\mathbf{S 6}$ (Figs. S6 and S7 in the supplementary material). The spectrum presents a peak at $953.09 \mathrm{~m} / \mathrm{z}$ with an isotopic mass distribution of 14 chlorine atoms, typical for PTM radical derivatives, that corresponds to the molecular ion of dyad $\mathbf{1}$.

\section{ELECTRICAL PROPERTIES OF THE TTF-PTM RADICAL DYAD ON HOPG}

Finally, we investigated the electrical properties of the dropcast self-assembled thin films S1, S4, and S5 supported on the electrically conducting HOPG substrates. For such a purpose, the I-V characteristics of such films were measured by C-AFM at RT under atmospheric conditions. HOPG $\mid$ Molecule $\mid P t / I r$ junctions were produced by contacting the top of the drop-cast films on HOPG with Pt/Ir coated AFM tips, which operated as a counter electrode in the two-terminal junction. To correlate the electrical characteristics with specific morphological features, we first imaged with tapping mode AFM the topography of a region of the drop-cast HOPG surface. Then, the C-AFM tip was brought to contact on a chosen point of the surface under an applied force of $2-3 \mathrm{nN}$ (higher forces or potentials destroyed the sample). The I-V curves were acquired while sweeping the applied voltage from $-1.0 \mathrm{~V}$ to $+1.0 \mathrm{~V}$. In particular, almost 100 curves per sample were recorded on different regions using always two or three different surfaces. The charge transport characteristics measured across the nanometer-thick molecular films of S1, S4, and S5 sandwiched between Pt/Ir tips and HOPG surfaces are portrayed in Fig. 3(a). Uncoated HOPG substrates were also measured prior to the functionalized surfaces to confirm that an "Ohmic" behavior was obtained [Fig. 3(a), 
a)

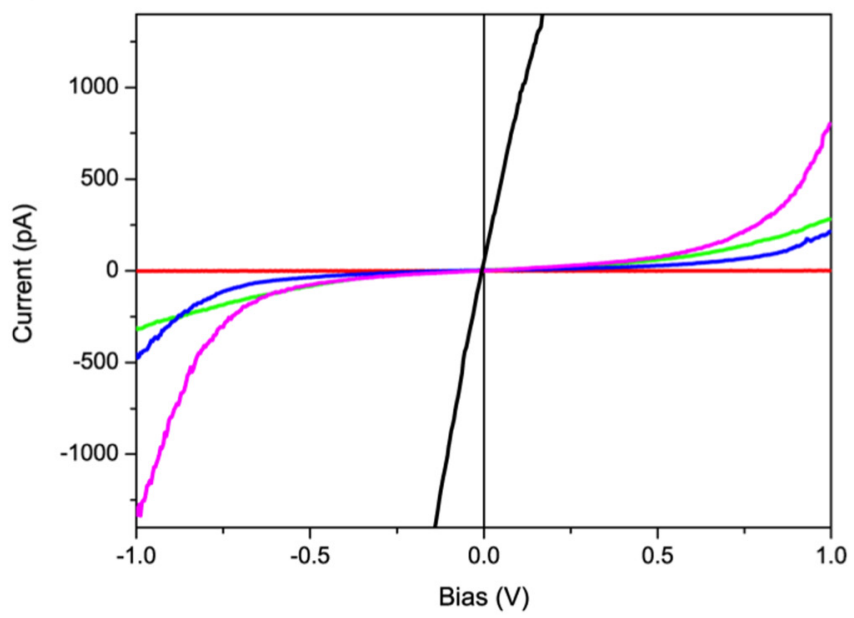

b)

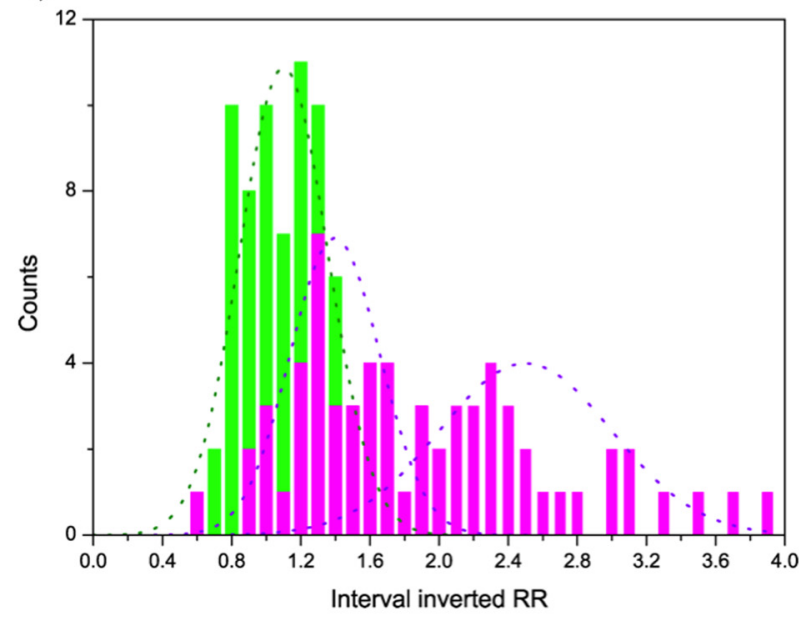

FIG. 3. (a) Representative I-V curves of pure HOPG (black line), $\mathbf{1} 1$ (blue and purple lines), $\mathbf{S 4}$ (red line, showing almost negligible currents), and $\mathbf{S 5}$ (green line) surfaces under an applied bias ranging from $-1.0 \mathrm{~V}$ to $+1.0 \mathrm{~V}$ using forces of 2-3 $\mathrm{nN}$. (b) Distributions of inverted rectification ratio values $[R R=\mathrm{J}(-1.0 \mathrm{~V}) / \mathrm{J}(+1.0 \mathrm{~V})]$ fitted with Gaussian curves (dotted lines), observed for S1 (purple bars) and S5 (green bars) surfaces. For S1 surfaces, two different RR distributions with mean values of 1.3 and 2.2 can be distinguished, while for S5, only one distribution centered at RR of 1.0 is observed. Unfortunately, the sensitivity of the employed measurement setup did not allow the determination of RR values for $\mathbf{S 4}$ surfaces.

black line]. S5 samples displayed symmetric I-V curves with currents of around $\pm 0.3 \mathrm{nA}$ at a maximum bias of $\pm 1.0 \mathrm{~V}$ (green line) as proved from all the 95 curves recorded in different regions of different samples. In contrast, $\mathbf{S} 4$ samples exhibited symmetric I-V curves with almost negligible currents of $\pm 5-10 \mathrm{pA}$ at the maximum bias of $\pm 1.0 \mathrm{~V}$. I-V curves measured in different areas of S1 samples showed two different behaviors, which could be explained by the presence of zones with different molecular orientations exhibiting distinct molecular surface charge transfers. Specifically, almost $70 \%$ of the I-V curves displayed currents of $\sim 1.3 \mathrm{nA}$, whereas the rest of the curves $(30 \%)$ showed more symmetric plots with currents of only $\sim 0.5 \mathrm{nA}$, in the negative bias (at $-1.0 \mathrm{~V}$ ). Thus, independent of the molecular orientation of the radical dyads on the surface, these data show that $\mathbf{S 1}$ surfaces have conductivities that are almost two orders of magnitude higher than those obtained with the non-radical dyad (S4). A similar enhancement of current was previously observed in two terminal junctions based on self-assembled monolayers (SAMs) covalently attached to gold metallic surfaces that contain the same TTF-PTM dyads. ${ }^{35}$ This large enhancement was attributed to the lower energy gap of the frontier orbitals (the SOMO, single occupied molecular orbital, and the SUMO, single unoccupied molecular orbital) of organic radicals in the SAMs when compared with the corresponding frontier orbitals of their non-radical counterparts (the HOMO and LUMO). As a result, the Fermi level of the electrodes is closer to the SUMO of the radical dyad than to the LUMO of the non-radical dyad, thereby enhancing notably the charge transport by a tunneling mechanism assisted by the SUMO orbital. ${ }^{35-39}$ Therefore, it is concluded that the enhancement of conductivity by the presence of radical molecules on metallic surfaces, named as the "radical-conductivity enhancement" phenomenon, does not require the organization of molecules in SAMs since it can be attained with a deposition by the easy and fast drop-casting method where molecules usually adopt random orientations.

It is also worth mentioning that all I-V curves obtained for $\mathbf{S 1}$ were slightly asymmetric, displaying small, but observable, inverted rectification values with larger currents at the negative bias than at the positive ones. Specifically, the curves with higher currents showed a mean inverted rectification ratio $[\mathrm{RR}=\mathrm{J}(-1.0 \mathrm{~V}) / \mathrm{J}(+1.0 \mathrm{~V})]$ of almost 1.3, whereas the ones with lower currents exhibited a mean value of 2.2 [Fig. 3(b)]. These mean rectification values are significant since S5 surfaces, measured under similar conditions, show the "radical-conductivity enhancement" phenomenon, but they do not exhibit any rectification. Similar small inverted behaviors were already reported in the literature for other dyads and ascribed to either a field-induced reorientation of the physisorbed molecules, the coexistence of phases with different molecular orientations, and/or polarisation-induced changes between the neutral and the zwitterionic ground states of the molecules in contact with the surface. ${ }^{40-45}$ Such explanations are also quite plausible for $\mathbf{S 1}$ because we used a drop-casting method that allows the molecules to freely orient with respect to the HOPG. It is also worth mentioning that a "Schottky" contribution to the small rectification of S1 cannot be completely ruled out due to the different work functions of the HOPG $(4.6 \mathrm{eV})$ and $\mathrm{Pt} / \mathrm{Ir}(5.2 \mathrm{eV})$ electrodes, although this electrode pair was also used for S5, where no rectification was observed.

\section{CONCLUSIONS}

In summary, solutions of radical dyad 1, non-radical dyad 2, and radical 3 were drop-cast on HOPG substrates employing different experimental conditions. The self-assembled nanostructures obtained on the surfaces from diluted solutions of DMF were 
characterized by ESR, CV, T-dependent XPS, and TOF-SIMs providing evidence that molecules remained intact upon physisorption on the surfaces. These surfaces were investigated by C-AFM showing that, in the case of the radical dyad $\mathbf{1}$, the molecules may adopt different molecular orientations exhibiting distinct molecular surface charge transfers that slightly alter the charge transport properties. This study also shows that the presence of PTM radical molecules notably enhances the electrical conduction and that this enhancement occurs in spite of the poor molecular orientation control achieved with the drop-casting technique. Moreover, the reported results demonstrate that the presence of a TTF subunit in the molecules can result in slightly asymmetric I-V curves. Thus, the results herein presented point out that PTM radical molecules with a functional group, drop-cast as thin films on surfaces, exhibit "radical-conductivity enhancement," which together with the functionality of the group attached to the radical unit, may bring new opportunities to the field of molecular electronics.

\section{EXPERIMENTAL METHODS}

All the glassware used in the preparation of films was washed with a $2 \% \mathrm{v} / \mathrm{v}$ Hellmanex II solution in Milli-Q water for $4 \mathrm{~h}$, rinsed, and dried at $150^{\circ} \mathrm{C}$ overnight. The HOPG substrates were used after mechanical cleavage with commercial Scotch ${ }^{\oplus}$ tape, whereas $\mathrm{Au}$ (111) substrates were rinsed with ethanol (HPLC grade) several times and dried under a nitrogen stream. The surfaces were prepared in a laminar flow cabinet under atmospheric conditions in the dark to protect dyad $\mathbf{1}$ and compound $\mathbf{3}$ from degradation. All the surfaces were prepared by drop-casting $2 \times 25 \mu \mathrm{l}$ of the desired solution using compounds synthesized as described elsewhere. ${ }^{20,46}$ Surfaces S1 to S5 were drop-cast with diluted solutions $(0.01 \mathrm{mM})$, whereas surface S6 was performed with a concentrated solution $(1 \mathrm{mM})$. The surfaces prepared using $\mathrm{CH}_{2} \mathrm{Cl}_{2}$ as a solvent were ready to measure after a couple of minutes of drop-casting the solution, while those prepared with toluene needed around $5 \mathrm{~h}$ to dry. When DMF was the solvent used, the surfaces were measured only after 2 days of being prepared. The surfaces were kept under atmospheric conditions once formed and no degradation was observed after 2 weeks.

\section{SUPPLEMENTARY MATERIAL}

See the supplementary material for technical information, the dewetting phenomenon of dyad $\mathbf{1}$ on HOPG, the nanostructures height analysis of S1-S3, the ESR spectroscopy and cyclic voltammetry of S1 on HOPG, the temperature dependent XPS spectra of S1 on HOPG, the temperature dependent ESR spectra of dyad 1 in bulk, and the TOF-SIMS of S6.

\section{ACKNOWLEDGMENTS}

We would like to acknowledge Raúl Pérez and Vega Lloveras for performing the TOF-SIMs and ESR measurements at the Scientific Park of Barcelona (Spain) and at ICMAB-CSIC, respectively. This work was supported by the Spanish MINECO/FEDER (Grant Nos. MAT 2016-80826-R and CTQ 2016-80030-R), Generalitat de Catalunya (Grant No. 2017-SGR 918 and CERCA Programme), CIBER-BBN, and the EU Marie Sklodowska-Curie
ITN project iSwitch (No. GA-642196). ICMAB is supported by the Spanish Ministry of Economy and Competitiveness, through the "Severo Ochoa" Programme for Centres of Excellence in R\&D (No. SEV-2015-0496). P.S. acknowledges financial support from Agence Nationale de la Recherche through the LabEx project Chemistry of Complex Systems (No. ANR-10-LABX-0026_CSC) and the International Center for Frontier Research in Chemistry (icFRC). J.G. is grateful to CSIC for a predoctoral JAE fellowship and M.S. to the Spanish Ministry of Education, Culture and Sports for an FPU.

\section{REFERENCES}

${ }^{1}$ J. M. Tour, Molecular Electronics: Commercial Insights, Chemistry, Devices, Architectures and Programming (World Scientific Publishing Co. Pte. Ltd, Singapore, 2003).

${ }^{2}$ R. M. Metzger, Topics in Current Chemistry (Springer, Heidelberg, 2012), Vol. 312.

${ }^{3}$ A. Aviram and M. A. Ratner, Chem. Phys. Lett. 27, 277-283 (1974).

${ }^{4}$ J. G. Ashwell, J. R. Sambles, A. S. Martin, W. G. Parker, and M. Szablewski, J. Chem. Soc. Chem. Commun. 19, 1374-1376 (1990).

${ }^{5}$ A. S. Martin, J. R. Sambles, and G. J. Ashwell, Phys. Rev. Lett. 70(2), 218-221 (1993).

${ }^{6}$ R. M. Metzger, B. Chen, U. Höpfner, M. V. Lakshmikantham, D. Vuillaume, T. Kawai, X. Wu, H. Tachibana, T. V. Hughes, H. Sakurai, J. W. Baldwin, C. Hosch, M. P. Cava, L. Brehmer, and G. J. Ashwell, J. Am. Chem. Soc. 119, 10455-10466 (1997).

${ }^{7}$ T. Xu, I. R. Peterson, M. V. Lakshmikantham, and R. M. Metzger, Angew. Chem. Int. Ed. 40(9), 1749-1752 (2001).

${ }^{8}$ R. M. Metzger, T. Xu, and I. R. Peterson, J. Phys. Chem. B 105, 7280-7290 (2001)

${ }^{\mathbf{9}}$ R. M. Metzger, Synth. Met. 159, 2277-2281 (2009).

${ }^{10}$ R. M. Metzger, Chem. Phys. 326, 176-187 (2006).

${ }^{11}$ G. Ho, J. R. Heath, M. Kondratenko, D. F. Perepichka, K. Arseneault, M. Pézolet, and M. R. Bryce, Chem. Eur. J. 11, 2914-2922 (2005).

${ }^{12}$ A. Honciuc, R. M. Metzger, A. Gong, and C. W. Spangler, J. Am. Chem. Soc. 129, 8310-8319 (2007).

${ }^{13}$ M. L. Chabinyc, X. Chen, R. E. Holmlin, H. Jacobs, H. Skulason, C. D. Frisbie, V. Mujica, M. A. Ratner, M. A. Rampi, and G. M. Whitesides, J. Am. Chem. Soc. 124, 11730 (2002).

${ }^{14}$ W. Schottky, Naturwissenschaften 26(52), 843 (1938).

${ }^{15}$ V. Palermo and P. Samorì, Angew. Chem. Int. Ed. 46, 4428-4432 (2007).

${ }^{16}$ K. Müllen and J. P. Rabe, Acc. Chem. Res. 41(4), 511-520 (2008).

17. A. Mann and W. R. Dichtel, J. Phys. Chem. Lett. 4, 2649-2657 (2013).

${ }^{18}$ G. Bottari, D. Olea, C. Gómez-Navarro, F. Zamora, J. Gómez-Herrero, and T. Torres, Angew. Chem. Int. Ed. 47, 2026-2031 (2008).

${ }^{19}$ T. W. Kelley, E. L. Granstrom, and C. D. Frisbie, Adv. Mater. 11(3), 261-264 (1999).

${ }^{20}$ J. Guasch, L. Grisanti, V. Lloveras, J. Vidal-Gancedo, M. Souto, D. C. Morales, M. Vilaseca, C. Sissa, A. Painelli, I. Ratera, C. Rovira, and J. Veciana, Angew. Chem. Int. Ed. 51, 11024-11028 (2012).

${ }^{21}$ J. Guasch, X. Fontrodona, I. Ratera, C. Rovira, and J. Veciana, Acta Crystallogr. C69, 255-257 (2013).

${ }^{22}$ M. Souto, C. Rovira, I. Ratera, and J. Veciana, CrystEngComm 19, 197-206 (2017).

${ }^{23}$ J. Guasch, L. Grisanti, M. Souto, V. Lloveras, J. Vidal-Gancedo, I. Ratera, A. Painelli, C. Rovira, and J. Veciana, J. Am. Chem. Soc. 135, 6958-6967 (2013).

${ }^{24}$ M. Souto, J. Guasch, V. Lloveras, P. Mayorga, J. T. L. E. Navarrete, J. Casado, I. Ratera, C. Rovira, A. Painelli, and J. Veciana, J. Phys. Chem. Lett. 4, 2721-2726 (2013).

${ }^{25}$ M. Cavallini and F. Biscarini, Nano Lett. 3(9), 1269-1271 (2003). 
${ }^{\mathbf{2 6}}$ J. Gómez-Segura, O. Kazakova, J. Davies, P. Josephs-Franks, J. Veciana, and D. Ruiz-Molina, Chem. Commun. 0, 5615-5617 (2005).

${ }^{27}$ C. A. Hunter and J. K. M. Sanders, J. Am. Chem. Soc. 112(14), 5525-5534 (1990).

${ }^{28}$ N. Crivillers, S. Furukawa, A. Minoia, A. V. Heyen, M. Mas-Torrent, C. Sporer, M. Linares, A. Volodin, C. V. Haesendonck, M. Van der Auweraer, R. Lazzaroni, S. D. Feyter, J. Veciana, and C. Rovira, J. Am. Chem. Soc. 131(17), 6246-6252 (2009).

${ }^{29}$ Q. Tang, Z. Zhou, and Z. Chen, J. Phys. Chem. C 115, 18531-18537 (2011).

${ }^{30}$ I. Ratera, D. Ruiz-Molina, F. Renz, J. Ensling, K. Wurst, C. Rovira, P. Gütlich, and J. Veciana, J. Am. Chem. Soc. 125(6), 1462-1463 (2003).

${ }^{31}$ G. D'Avino, L. Grisanti, J. Guasch, I. Ratera, J. Veciana, and A. Painelli, J. Am. Chem. Soc. 130, 12064-12072 (2008).

${ }^{32}$ L. Grisanti, G. D'Avino, A. Painelli, J. Guasch, I. Ratera, and J. Veciana, J. Phys. Chem. B 113(14), 4718-4725 (2009).

${ }^{33}$ G. D'Avino, L. Grisanti, A. Painelli, J. Guasch, I. Ratera, and J. Veciana, CrystEngComm 11, 2040-2047 (2009).

34 J. Guasch, L. Grisanti, S. Jung, D. Morales, G. D'Avino, M. Souto, X. Fontrodona, A. Painelli, F. Renz, I. Ratera, and J. Veciana, Chem. Mater. 25, 808-814 (2013).

${ }^{35}$ M. Souto, V. Díez-Cabanes, L. Yuan, A. Kyvik, I. Ratera, C. A. Nijhuis, J. Cornil, and J. Veciana, Phys. Chem. Chem. Phys. 20, 25638-25647 (2018).
${ }^{36}$ N. Crivillers, M. Paradinas, M. Mas-Torrent, S. T. Bromley, C. Rovira, C. Ocal, and J. Veciana, Chem. Commun. 47, 4664-4666 (2011).

${ }^{37}$ L. Yuan, C. Franco, N. Crivillers, M. Mas-Torrent, L. Cao, C. S. S. Sangeeth, C. Rovira, J. Veciana, and C. A. Nijhuis, Nat. Commun. 7, 12066 (2016).

${ }^{38} \mathrm{~F}$. Bejarano, I. J. Olavarria-Contreras, A. Droghetti, I. Rungger, A. Rudnev, D. Gutiérrez, M. Mas-Torrent, J. Veciana, H. S. J. V. D. Zant, C. Rovira, E. Burzurí, and N. Crivillers, J. Am. Chem. Soc. 140(5), 1691-1696 (2018).

${ }^{39}$ M. Souto, L. Yuan, D. C. Morales, L. Jiang, I. Ratera, C. A. Nijhuis, and J. Veciana, J. Am. Chem. Soc. 139(12), 4262-4265 (2017).

${ }^{40}$ G. J. Ashwell, W. D. Tyrrell, and A. J. Whittam, J. Mater. Chem. 13, 2855-2857 (2003).

${ }^{41}$ G. J. Ashwell, W. D. Tyrrell, and A. J. Whittam, J. Am. Chem. Soc. 126, 7102-7110 (2004).

${ }^{42}$ G. J. Ashwell, A. Chwialkowska, and L. R. H. High, J. Mater. Chem. 14, 2389-2394 (2004).

${ }^{43}$ G. J. Ashwell and A. Mohib, J. Am. Chem. Soc. 127, 16238-16244 (2005).

${ }^{44}$ G. J. Ashwell, B. J. Robinson, M. A. Amiri, D. Locatelli, S. Quicicde, and D. Roberto, J. Mater. Chem. 15, 4203-4205 (2005).

${ }^{45}$ G. J. Ashwell, B. Urasinska, and W. D. Tyrrell, Phys. Chem. Chem. Phys. 8, 3314-3319 (2006).

${ }^{46} \mathrm{M}$. Ballester, J. Riera, J. Castañer, C. Badía, and J. M. Monsó, J. Am. Chem. Soc. 93, 2215-2225 (1971). 